\title{
Energy cascade rate in isothermal compressible magnetohydrodynamic turbulence
}

\author{
N. Andrés ${ }^{1}$, F. Sahraoui ${ }^{1}$, S. Galtier ${ }^{1,2}$, L. Z. $\operatorname{Hadid}^{3}$, P. Dmitruk ${ }^{4,5}$, and P. D. Mininni ${ }^{4,5}$ \\ 1 Laboratoire de Physique des Plasmas, École Polytechnique, \\ CNRS, Sorbonne University, Observatoire de Paris, \\ Univ. Paris-Sud, F-91128 Palaiseau Cedex, France \\ 2 Univ. Paris-Sud, Université Paris-Saclay, France. \\ 3 Swedish Institute of Space Physics, Uppsala, Sweden. \\ 4 Departamento de Física, Facultad de Ciencias Exactas y Naturales, \\ Universidad de Buenos Aires, Ciudad Universitaria, 1428, Buenos Aires, Argentina. \\ 5 Instituto de Física de Buenos Aires, CONICET-UBA, \\ Ciudad Universitaria, 1428, Buenos Aires, Argentina.
}

\begin{abstract}
Three-dimensional direct numerical simulations are used to study the energy cascade rate in isothermal compressible magnetohydrodynamic turbulence. Our analysis is guided by a two-point exact law derived recently for this problem in which flux, source, hybrid, and mixed terms are present. The relative importance of each term is studied for different initial subsonic Mach numbers $M_{S}$ and different magnetic guide fields $\mathbf{B}_{0}$. The dominant contribution to the energy cascade rate comes from the compressible flux, which depends weakly on the magnetic guide field $\mathbf{B}_{0}$, unlike the other terms whose modulus increase significantly with $M_{S}$ and $\mathbf{B}_{0}$. In particular, for strong $\mathbf{B}_{0}$ the source and hybrid terms are dominant at small scales with almost the same amplitude but with a different sign. A statistical analysis made with an isotropic decomposition based on the $\mathrm{SO}(3)$ rotation group is shown to generate spurious results in presence of $\mathbf{B}_{0}$, when compared with an axisymmetric decomposition better suited to the geometry of the problem. Our numerical results are eventually compared with previous analyses made with in-situ measurements in the solar wind and the terrestrial magnetosheath.
\end{abstract}

\section{INTRODUCTION}

Exact results in fully developed turbulence represent strong boundary conditions that any model must satisfy [1], however there are only a few of such predictions. The so-called " $4 / 5$ law" is an exact relation for incompressible hydrodynamic (HD) turbulence. In the infinite Reynolds number limit and assuming space homogeneity, isotropy and time stationary, this law expresses how the two-point third-order structure function for the velocity field is connected to the energy cascade rate $\varepsilon$. In particular, in Fourier space this exact relation leads dimensionally to the well-known Kolmogorov energy spectrum $E_{k} \sim \varepsilon^{2 / 3} k^{-5 / 3}[2,3]$. For incompressible magnetohydrodynamic (IMHD) turbulence, Chandrasekhar [4] derived such an exact relation under the assumptions of infinite kinetic and magnetic Reynolds numbers, time stationarity, space homogeneity, and full isotropy (i.e., rotation and mirror symmetries). Later, Politano and Pouquet $[5,6]$ derived the so-called 4/3 law for IMHD turbulence, which gives a simple relation between two-point thirdorder structure functions, the distance between the two points, and the energy dissipation rate.

The validity of the exact law in IMHD turbulence has been the subject of several numerical tests [see, e.g. 7-10]. For example, Mininni and Pouquet [7] reported high spatial resolution results for decaying IMHD turbulence in which the energy dissipation rate seemed to reach asymptotically a constant value at large Reynolds numbers. An extension of the exact IMHD law in presence of a constant velocity shear was proposed and tested numerically with direct numerical simulations (DNS) of twodimensional (2D) IMHD [9]. Among several other uses, the exact laws for IMHD turbulence provides a precise identification of the inertial range [see, e.g., 11, and references therein], and an estimate of the energy cascade rate and the Reynolds numbers in experiments of turbulence, in particular when dissipation mechanisms are unknown such as in near-Earth space plasmas [12-15].

Under the classical assumptions of homogeneity, sta- 
tionarity, and infinite kinetic/magnetic Reynolds numbers, Banerjee and Galtier [16] derived an exact law for isothermal compressible MHD (CMHD) turbulence. Their results revealed the presence of a new type of term that acts in the inertial range as a source (or a sink) for the energy cascade rate [see also, 17]. It is worth noticing that in IMHD turbulence there is only one type of term, the flux, that transfers energy in the inertial range $[1,18]$. Because of its complexity, the expression of the exact law in CMHD is not unique [e.g., see 19]. For example, Andrés and Sahraoui [20] have re-derived the law using the plasma velocity, the compressible Alfvén velocity, and the plasma density as primitive variables. The authors found four different categories of terms that are involved in the inertial range. Besides the flux and the sources previously reported, the authors also found two new types of terms to which they referred to as hybrid and $\beta$-dependent terms (with $\beta$ the ratio between the plasma and magnetic pressure). It is the main goal of the present paper to investigate numerically the relative importance and the contribution of each of these terms to the exact law in CMHD isothermal turbulence.

The role of density fluctuations in the solar wind energy cascade rate was investigated by Carbone et al. [21]. Using Ulysses solar wind data the authors found a better scaling relation with a heuristic compressible model than with the IMHD exact relation, showing therefore the relevance of density fluctuations in the cascade process (see a discussion of this model in Hadid et al. [22]). Following a more rigorous approach, Banerjee et al. [23] used the exact law for isothermal CMHD [16] to analyze the fast solar wind data from the THEMIS mission. The authors performed a term-by-term analysis, showed the existence of an inertial range over more than two decades of scales, and found that the compressible fluctuations increase (from 2 to 4 times) the estimation of the turbulent cascade rate with respect to the estimations stemming from the incompressible model. Hadid et al. [22] extended the previous analysis (still using THEMIS data) to the slow solar wind which is known to be more compressible. In this case they found that the compressible energy cascade rate is increased even further (because of higher density fluctuations in the slow solar wind when compared to the fast wind) and that it obeys a powerlaw scaling with the turbulent Mach number. However, it is worth noticing that in all these recent studies [2224] several source terms of the exact CMHD law have been neglected. It is the goal of the present paper to check carefully if the assumptions made to neglect these terms are indeed satisfied in DNS close to the solar wind conditions.

Recently, several new results have been obtained in compressible turbulence that are worth mentioning here. For example, Zank et al. [25] used the nearly incompressible MHD (NI MHD) equations [e.g., see 26] to describe solar wind homogeneous or inhomogeneous turbulence for plasma $\beta \lesssim 1$. The authors presented a NI MHD formulation describing the transport throughout the solar wind of turbulence which was in its majority 2D, and with a small slab component. Using Voyager 1 measurements, Zank et al. [27] showed that inner heliosheath fast and slow MHD waves incident on the heliopause generate, in the very local interstellar medium (LISM), only fast MHD waves that propagate into this medium. The authors suggested that this may be the origin of compressible turbulence in the LISM.

On the other hand, Yang et al. [28] used DNS of mechanically forced CMHD turbulence to study the degree to which some turbulence theories proposed for incompressible flows remain applicable in the compressible case. In particular, intermittency, coherent structures, and energy cascade rates were studied with different forcing mechanisms. Grete et al. [29] extended the classical shell-to-shell energy transfer analysis to the isothermal compressible regime. The authors derived four new transfer functions in order to measure, e.g., the energy exchange via the magnetic pressure. Andrés et al. [30] showed direct numerical evidence of the excitation of magnetosonic and Alfvén waves in three-dimensional (3D) CMHD turbulence at small sonic Mach numbers. Using spatio-temporal spectra, in the low $\beta$ regime, the authors found excitation of compressible and incompressible fluctuations, with a clear transfer of energy towards Alfvénic and 2D modes. However, in the high $\beta$ regime, fast and slow magnetosonic waves were present with no clear signature of Alfvén waves, a significant part of the energy being carried by 2D turbulent eddies. Finally, Andrés et al. [31] have derived an exact law for 3D homogeneous compressible isothermal Hall magnetohydrodynamic turbulence, without the assumption of isotropy. 
The authors showed that the Hall current introduces new flux and source terms that act at the small scales (comparable or smaller than the ion skin depth) to significantly impact the turbulence dynamics.

The main goal of the present paper is thus to investigate the energy cascade rate in isothermal CMHD turbulence using 3D DNS. We present a comprehensive analysis of the exact law, with a particular emphasis on the nature of each term involved in the nonlinear cascade of energy, and on the role of the background magnetic field $\mathbf{B}_{0}$. Furthermore, we discuss our numerical results in the context of the original observational results from Refs. [22, 23]. We expect that our numerical findings will help to clarify some subtle issues regarding the use of the compressible exact law in DNS and spacecraft data.

The paper is organized as follows: in Sec. II A we describe the CMHD equations; in Sec. II B we present the exact law for fully developed isothermal CMHD turbulence; in Sec. II C and IID we introduce the numerical code and techniques used to compute the different correlation functions; in Sec. III we expose our numerical results and, finally, in Sec. IV we discuss the main findings and their implications for the observational studies in the near-Earth space.

\section{THEORY}

\section{A. Compressible MHD}

The 3D CMHD equations correspond to the continuity equation for the mass density, the momentum equation for the velocity field in which the Lorentz force is included, the induction equation for the magnetic field, and the differential Gauss' law. These equations can be written as [see, e.g., 30, 32],

$$
\begin{aligned}
& \frac{\partial \rho}{\partial t}=-\boldsymbol{\nabla} \cdot(\rho \mathbf{u}) \\
& \frac{\partial \mathbf{u}}{\partial t}=-\mathbf{u} \cdot \nabla \mathbf{u}-\frac{\nabla P}{\rho}+\frac{(\boldsymbol{\nabla} \times \mathbf{B}) \times \mathbf{B}}{4 \pi \rho}+\mathbf{f}_{k}+\mathbf{d}_{k} \\
& \frac{\partial \mathbf{B}}{\partial t}=\boldsymbol{\nabla} \times(\mathbf{u} \times \mathbf{B})+\mathbf{f}_{m}+\mathbf{d}_{m} \\
& \boldsymbol{\nabla} \cdot \mathbf{B}=0
\end{aligned}
$$

where $\mathbf{u}$ is the velocity field fluctuation, $\mathbf{B}=\mathbf{B}_{0}+\mathbf{b}$ is the total magnetic field, $\rho$ is the mass density, and $P$ is the scalar pressure. For the sake of simplicity we assume that the plasma follows an isothermal equation of state, $P=$ $c_{s}^{2} \rho$, where $c_{s}$ is the constant sound speed, which allows us to close the hierarchy of the fluid equations (no energy equation is further needed). Finally, $\mathbf{f}_{k, m}$ are respectively a mechanical and the curl of the electromotive large-scale forcings, and $\mathbf{d}_{k, m}$ are respectively the small-scale kinetic and magnetic dissipation terms.

Alternatively to the magnetic field $\mathbf{B}$, the compressible Alfvén velocity $\mathbf{u}_{\mathrm{A}} \equiv \mathbf{B} / \sqrt{4 \pi \rho}$ can be used (where $\rho$ is time and space dependent). In this manner, both field variables, $\mathbf{u}$ and $\mathbf{u}_{\mathrm{A}}$, are expressed in speed units. Therefore, Eqs. (1)-(4) can be cast as [33],

$$
\begin{aligned}
\frac{\partial e}{\partial t}= & -\mathbf{u} \cdot \nabla e-c_{s}^{2} \boldsymbol{\nabla} \cdot \mathbf{u} \\
\frac{\partial \mathbf{u}}{\partial t}= & -\mathbf{u} \cdot \nabla \mathbf{u}+\mathbf{u}_{\mathrm{A}} \cdot \nabla \mathbf{u}_{\mathrm{A}}-\frac{1}{\rho} \boldsymbol{\nabla}\left(P+P_{M}\right) \\
& -\mathbf{u}_{\mathrm{A}}\left(\boldsymbol{\nabla} \cdot \mathbf{u}_{\mathrm{A}}\right)+\mathbf{f}_{k}+\mathbf{d}_{k} \\
\frac{\partial \mathbf{u}_{\mathrm{A}}}{\partial t}= & -\mathbf{u} \cdot \nabla \mathbf{u}_{\mathrm{A}}+\mathbf{u}_{\mathrm{A}} \cdot \nabla \mathbf{u}-\frac{\mathbf{u}_{\mathrm{A}}}{2}(\boldsymbol{\nabla} \cdot \mathbf{u})+\mathbf{f}_{m}+\mathbf{d}_{m}
\end{aligned}
$$

$\mathbf{u}_{\mathrm{A}} \cdot \nabla \rho=-2 \rho\left(\boldsymbol{\nabla} \cdot \mathbf{u}_{\mathrm{A}}\right)$,

where $P_{M} \equiv \rho u_{\mathrm{A}}^{2} / 2$ is the magnetic pressure. Note that we have written Eq. (3) as a function of the internal compressible energy for an isothermal plasma, i.e., $e \equiv$ $c_{s}^{2} \ln \left(\rho / \rho_{0}\right)$, where $\rho_{0}$ is a constant (of reference) mass density. In the rest of the paper we shall assume that the fields considered are regular and therefore differentiable. Singular fields may exist in the inviscid case, leading to the appearance of anomalous dissipation [34-36].

\section{B. Exact law for CMHD turbulence}

Following the usual assumptions for fully developed homogeneous turbulence (i.e., infinite kinetic and magnetic Reynolds numbers and a steady state with a balance between forcing and dissipation $[11,16,17,37])$, an exact law for CMHD turbulence can be obtained as [16, 20], 


$$
\begin{aligned}
-2 \varepsilon_{C}= & \frac{1}{2} \boldsymbol{\nabla}_{r} \cdot\left\langle\left[\left(\delta(\rho \mathbf{u}) \cdot \delta \mathbf{u}+\delta\left(\rho \mathbf{u}_{\mathrm{A}}\right) \cdot \delta \mathbf{u}_{\mathrm{A}}+2 \delta e \delta \rho\right] \delta \mathbf{u}-\left[\delta(\rho \mathbf{u}) \cdot \delta \mathbf{u}_{\mathrm{A}}+\delta \mathbf{u} \cdot \delta\left(\rho \mathbf{u}_{\mathrm{A}}\right)\right] \delta \mathbf{u}_{\mathrm{A}}\right\rangle\right. \\
& +\left\langle\left[R_{E}^{\prime}-\frac{1}{2}\left(R_{B}^{\prime}+R_{B}\right)-E^{\prime}+\frac{P_{M}^{\prime}-P^{\prime}}{2}\right](\boldsymbol{\nabla} \cdot \mathbf{u})+\left[R_{E}-\frac{1}{2}\left(R_{B}+R_{B}^{\prime}\right)-E+\frac{P_{M}-P}{2}\right]\left(\boldsymbol{\nabla}^{\prime} \cdot \mathbf{u}^{\prime}\right)\right\rangle \\
& +\left\langle\left[\left(R_{H}-R_{H}^{\prime}\right)-\bar{\rho}\left(\mathbf{u}^{\prime} \cdot \mathbf{u}_{\mathrm{A}}\right)+H^{\prime}\right]\left(\boldsymbol{\nabla} \cdot \mathbf{u}_{\mathrm{A}}\right)+\left[\left(R_{H}^{\prime}-R_{H}\right)-\bar{\rho}\left(\mathbf{u} \cdot \mathbf{u}_{\mathrm{A}}^{\prime}\right)+H\right]\left(\boldsymbol{\nabla}^{\prime} \cdot \mathbf{u}_{\mathrm{A}}^{\prime}\right)\right\rangle \\
& +\frac{1}{2}\left\langle\left(e^{\prime}+{\frac{u_{\mathrm{A}}}{2}}^{2}\right)[\boldsymbol{\nabla} \cdot(\rho \mathbf{u})]+\left(e+{\frac{u_{\mathrm{A}}}{2}}^{2}\right)\left[\boldsymbol{\nabla}^{\prime} \cdot\left(\rho^{\prime} \mathbf{u}^{\prime}\right)\right]\right\rangle \\
& -\frac{1}{2}\left\langle\beta^{-1^{\prime}} \boldsymbol{\nabla}^{\prime} \cdot\left(e^{\prime} \rho \mathbf{u}\right)+\beta^{-1} \boldsymbol{\nabla} \cdot\left(e \rho^{\prime} \mathbf{u}^{\prime}\right)\right\rangle
\end{aligned}
$$

where $\varepsilon_{C}$ is the total compressible energy cascade rate. We have defined the total energy and the densityweighted cross-helicity respectively as

$$
\begin{aligned}
E(\mathbf{x}) & \equiv \frac{\rho}{2}\left(\mathbf{u} \cdot \mathbf{u}+\mathbf{u}_{\mathrm{A}} \cdot \mathbf{u}_{\mathrm{A}}\right)+\rho e \\
H(\mathbf{x}) & \equiv \rho\left(\mathbf{u} \cdot \mathbf{u}_{\mathrm{A}}\right)
\end{aligned}
$$

and their associated two-point correlation functions as,

$$
\begin{aligned}
& R_{E}\left(\mathbf{x}, \mathbf{x}^{\prime}\right) \equiv \frac{\rho}{2}\left(\mathbf{u} \cdot \mathbf{u}^{\prime}+\mathbf{u}_{\mathrm{A}} \cdot \mathbf{u}_{\mathrm{A}}^{\prime}\right)+\rho e^{\prime} \\
& R_{H}\left(\mathbf{x}, \mathbf{x}^{\prime}\right) \equiv \frac{\rho}{2}\left(\mathbf{u} \cdot \mathbf{u}_{\mathrm{A}}^{\prime}+\mathbf{u}_{\mathrm{A}} \cdot \mathbf{u}^{\prime}\right)
\end{aligned}
$$

In addition, we have defined the magnetic energy density $R_{B}\left(\mathbf{x}, \mathbf{x}^{\prime}\right) \equiv \rho\left(\mathbf{u}_{\mathrm{A}} \cdot \mathbf{u}_{\mathrm{A}}^{\prime}\right) / 2$, the prime denotes field evaluation at $\mathbf{x}^{\prime}=\mathbf{x}+\boldsymbol{\ell}(\boldsymbol{\ell}$ being the displacement vector) and the angular bracket $\langle\cdot\rangle$ denotes an ensemble average. It is worth mentioning that the properties of spatial homogeneity implies (assuming ergodicity) that the results of averaging over a large number of realizations can be obtained equally well by averaging over a large region of space for one realization [38]. Finally, we have introduced the usual increments and local mean definitions, i.e. $\delta \alpha \equiv \alpha^{\prime}-\alpha$ and $\bar{\alpha} \equiv\left(\alpha^{\prime}+\alpha\right) / 2$ (with $\alpha$ any scalar function), respectively.

We recall that the derivation of the exact law (9) does not require the assumption of isotropy and that it is independent of the dissipation mechanisms acting in the plasma (assuming that the dissipation acts only at the smallest scales in the system) [see also, 11, 17, 37]. In a compact form, the exact law for CMHD turbulence (i.e., Eq. 9) can be schematically written as,

$$
-2 \varepsilon_{C}=\frac{1}{2} \nabla_{\ell} \cdot \mathbf{F}_{\mathrm{C}}+\mathrm{S}_{\mathrm{C}}+\mathrm{S}_{\mathrm{H}}+\mathrm{M}_{\beta}
$$

where $\mathbf{F}_{\mathrm{C}}, \mathrm{S}_{\mathrm{C}}, \mathrm{S}_{\mathrm{H}}$ and $\mathrm{M}_{\beta}$ represent the total compressible flux, source, hybrid and $\beta$-dependent terms, respectively, with by definition

$$
\begin{aligned}
\mathbf{F}_{\mathrm{C}} \equiv & \left\langle\left[\left(\delta(\rho \mathbf{u}) \cdot \delta \mathbf{u}+\delta\left(\rho \mathbf{u}_{\mathrm{A}}\right) \cdot \delta \mathbf{u}_{\mathrm{A}}+2 \delta e \delta \rho\right] \delta \mathbf{u}-\left[\delta(\rho \mathbf{u}) \cdot \delta \mathbf{u}_{\mathrm{A}}+\delta \mathbf{u} \cdot \delta\left(\rho \mathbf{u}_{\mathrm{A}}\right)\right] \delta \mathbf{u}_{\mathrm{A}}\right\rangle,\right. \\
\mathrm{S}_{\mathrm{C}} \equiv & \left\langle\left[R_{E}^{\prime}-\frac{1}{2}\left(R_{B}^{\prime}+R_{B}\right)\right](\boldsymbol{\nabla} \cdot \mathbf{u})+\left[R_{E}-\frac{1}{2}\left(R_{B}+R_{B}^{\prime}\right)\right]\left(\boldsymbol{\nabla}^{\prime} \cdot \mathbf{u}^{\prime}\right)\right\rangle \\
& +\left\langle\left[\left(R_{H}-R_{H}^{\prime}\right)-\bar{\rho}\left(\mathbf{u}^{\prime} \cdot \mathbf{u}_{\mathrm{A}}\right)\right]\left(\boldsymbol{\nabla} \cdot \mathbf{u}_{\mathrm{A}}\right)+\left[\left(R_{H}^{\prime}-R_{H}\right)-\bar{\rho}\left(\mathbf{u} \cdot \mathbf{u}_{\mathrm{A}}^{\prime}\right)\right]\left(\boldsymbol{\nabla}^{\prime} \cdot \mathbf{u}_{\mathrm{A}}^{\prime}\right)\right\rangle, \\
\mathrm{S}_{\mathrm{H}} \equiv & \left\langle\left(\frac{P_{M}^{\prime}-P^{\prime}}{2}-E^{\prime}\right)(\boldsymbol{\nabla} \cdot \mathbf{u})+\left(\frac{P_{M}-P}{2}-E\right)\left(\boldsymbol{\nabla}^{\prime} \cdot \mathbf{u}^{\prime}\right)\right\rangle+\left\langle H^{\prime}\left(\boldsymbol{\nabla} \cdot \mathbf{u}_{\mathrm{A}}\right)+H\left(\boldsymbol{\nabla}^{\prime} \cdot \mathbf{u}_{\mathrm{A}}^{\prime}\right)\right\rangle \\
& +\frac{1}{2}\left\langle\left(e^{\prime}+{\frac{u_{\mathrm{A}}}{2}}^{\prime}\right)[\boldsymbol{\nabla} \cdot(\rho \mathbf{u})]+\left(e+{\frac{u_{\mathrm{A}}}{2}}^{2}\right)\left[\nabla^{\prime} \cdot\left(\rho^{\prime} \mathbf{u}^{\prime}\right)\right]\right\rangle, \\
\mathrm{M}_{\beta} \equiv & -\frac{1}{2}\left\langle\beta^{-1^{\prime}} \boldsymbol{\nabla}^{\prime} \cdot\left(e^{\prime} \rho \mathbf{u}\right)+\beta^{-1} \boldsymbol{\nabla} \cdot\left(e \rho^{\prime} \mathbf{u}^{\prime}\right)\right\rangle .
\end{aligned}
$$

Eq. (15) is the energy flux, which is the usual term present in the exact law of incompressible turbulence [20]. 
This term is written as a global divergence of products of increments of different variables. It is worth mentioning that the total compressible flux (15) is a combination of fourth- and third-order terms, which makes a major difference with the incompressible case where the flux terms are usually third-order correlation functions. The appearance of a fourth-order correlation function is a direct consequence of the total energy definition in the CMHD model (see Eq. 10). The purely compressible source terms in Eq. (16) may act as a source (or a sink) for the mean energy cascade rate in the inertial range. These terms involve two-point correlation functions (namely $R_{E}, R_{B}$ and $R_{H}$ ) and are proportional to the divergence of the Alfvén and kinetic velocity fields.

The hybrid term offers the freedom to be written either as a flux- or as a source-like term. However, when written as a flux-like term it cannot be expressed as the product of increments, as the usual flux in incompressible HD and MHD turbulence [2-6, 39] or the flux term in Eq. 15. On the other hand, the mixed $\beta$-dependent term (already reported as a flux-like term in Banerjee and Galtier [16] under some condition) has no counterpart in compressible HD turbulence $[17,40]$ and cannot, in general, be expressed either as purely flux or a source. Besides, the mixed $\beta$-dependent term stems from the magnetic pressure gradient term in the momentum equation (1).

The schematic representation (14) reflects the true nature of each term in the exact law for CMHD turbulence [20]. In order to understand the impact of each contribution in the nonlinear energy cascade rate, we have used this particular organization. It is worth mentioning that in the observational works of Refs. [22, 23], $\mathrm{F}_{\mathrm{C}}$, part of $\mathrm{M}_{\beta}$ (under the assumption of statistical stationarity of the $\beta$ parameter), and part of $\mathrm{S}_{\mathrm{H}}$ were considered in the evaluation of the solar wind energy cascade rate. The remaining terms were considered as sources and assumed to be sub-dominant in the inertial range [see, 41]. We will return to this issue in Sec. IV.

Integrating Eq. (14) over a cylinder of radius $\ell_{\perp}$, we can obtain an approximate scalar relation for anisotropic turbulence in a symbolic form (assuming that perpendicular correlation functions do not depend in $\ell_{\|}$and parallel correlation functions do not depend in $\ell_{\perp}$ ),

$$
-4 \varepsilon_{C} \ell_{\perp}=\mathrm{F}_{\mathrm{C}}+\mathrm{Q}_{\mathrm{S}_{\mathrm{C}}}+\mathrm{Q}_{\mathrm{S}_{\mathrm{H}}}+\mathrm{Q}_{\mathrm{M}_{\beta}}
$$

\begin{tabular}{lllll}
\hline \hline Run & $B_{0}$ & $M_{S}$ & \multicolumn{2}{c}{$\left\langle\delta E_{u}\right\rangle\left\langle\delta E_{b}\right\rangle$} \\
\hline I & 0 & $1 / 4$ & 0.13 & 0.14 \\
II & 2 & $1 / 4$ & 0.15 & 0.05 \\
III & 8 & $1 / 4$ & 0.16 & 0.06 \\
IV & 0 & $1 / 2$ & 0.13 & 0.14 \\
\hline \hline
\end{tabular}

Table I. Parameters used in Runs I to IV: $B_{0}$ is the magnetic guide field, $M_{S}$ is the sonic Mach number, $\left\langle\delta E_{u}\right\rangle$ and $\left\langle\delta E_{B}\right\rangle$ are the average fluctuating kinetic and magnetic energies reached in the stationary state.

where $\mathrm{F}_{\mathrm{C}} \equiv \mathbf{F}_{\mathrm{C}} \cdot \boldsymbol{\ell}_{\perp} / \ell_{\perp}$ and the integral functions correspond to

$$
\mathrm{Q}_{\mathrm{T}} \equiv \frac{2}{\ell_{\perp}} \int_{0}^{\ell_{\perp}} \mathrm{T}\left(\ell_{\perp}^{*}\right) \ell_{\perp}^{*} d \ell_{\perp}^{*},
$$

with $\mathrm{T}\left(\ell_{\perp}\right)=\mathrm{S}_{\mathrm{C}}\left(\ell_{\perp}\right), \mathrm{S}_{\mathrm{H}}\left(\ell_{\perp}\right)$ and $\mathrm{M}_{\beta}\left(\ell_{\perp}\right)$, respectively.

\section{Numerical code}

The 3D CMHD Eqs. (1)-(4) are numerically solved using the Fourier pseudo-spectral code GHOST [42, 43] with a new module for compressible flows based on previous developments $[44,45]$. The numerical scheme used ensures the exact energy conservation for the continuous time spatially discrete equations [43] (as well as conservation of all other quadratic invariants in the system). We used a linear spatial resolution of $N=512$ grid points in each direction in a cubic periodic box. For simplicity, we used identical dimensionless viscosity and magnetic diffusivity, $\nu=\eta=1.25 \times 10^{-3}$ (i.e., the magnetic Prandtl number is one). In all our runs, $k_{\text {min }}=1$ for a box of length $L_{0}=2 \pi$ and $N=512$ leads to a maximum wavenumber $k_{\max }=N / 3 \sim 170$ (de-aliasing rule). At all times, we have checked that $k_{D} / k_{\max }<1, k_{D}$ being the dissipation wave number.

The initial state of our simulations corresponds to density, velocity and magnetic fields amplitude fluctuations equal to zero. For all times $t>0$, the velocity field and the magnetic vector potential are forced by a solenoidal mechanical and an electromotive forcing, respectively, at the largest scales of the numerical box (i.e., $1 \leq k_{f} \leq 3$ ). The mechanical and electromotive forcings are random and uncorrelated, and they inject neither kinetic nor 
magnetic helicity. Furthermore, the set of random phases of the two forces are independent. Random phases were also slowly evolving in time, to avoid introducing longterm correlations, but also to prevent introducing very fast time scales. To this end, a new set of random phases is generated for each forcing function every $1 / 2$ turnover time. Finally, the forcing are linearly interpolated from their previous states to the new random states on $1 / 2$ turnover time, and the process is then repeated (for more details about the random forcing used here, see [30]). We performed four numerical simulations with initial subsonic Mach numbers $M_{S}=u_{0} / c_{s}$ (tipically, $u_{0} \sim 1$ ) and with different background magnetic field $B_{0}$ (see Table I). This allows us to investigate different regimes of CMHD turbulence, with a special emphasis on the magnetic guide field and the level of compressibility of the plasma. In all cases studied here $\mathbf{B}_{0}$ is along the $\hat{\mathbf{z}}$ axis.

\section{Correlation functions}

For the computation of correlation functions in multiple directions (and thus to increase statistical convergence by averaging over all these directions), we use the angle-averaged technique presented in Taylor et al. [46]. This technique avoids the need to use 3D interpolations to compute the correlation functions in directions for which the evaluation points do not lie on grid points. This significantly reduces the computational cost of any geometrical decomposition of the flow [47]. In particular, and considering that we have simulations without and with a magnetic guide field, for which we can expect the fields to be respectively statistically isotropic or axisymmetric, we have used two decompositions: the one based on the $\mathrm{SO}(3)$ rotation group for isotropic turbulence, and another one based on the $\mathrm{SO}(2) \times \mathrm{R}$ symmetry group (i.e., rotations in the $\hat{\mathbf{x}}-\hat{\mathbf{y}}$ plane plus translations in the $\hat{\mathbf{z}}$ direction) for anisotropic (axisymmetric) turbulence.

The procedure used to average each term in Eqs. (9) over several directions can be summarized as follows: in the isotropic SO(3) decomposition, the correlation functions are computed along different directions generated by the vectors (all are in units of grid points in the simulation box) $(1,0,0),(1,1,0),(1,1,1),(2,1,0),(2,1,1),(2,2,1)$,
$(3,1,0),(3,1,1)$ and taking all the index and sign permutations of the three coordinates (and removing any vector that is a positive or negative multiple of any other vector in the set) $[46,48]$. This procedure generates 73 unique directions. In this manner, the $\mathrm{SO}(3)$ decomposition gives the correlation functions as a function of 73 radial directions covering almost all the sphere [46], whose averaging results in the isotropic correlation functions that depend on $\ell$.

In the $\mathrm{SO}(2)$ case, the correlation functions are computed using 12 different directions generated by integer multiples of the vectors $(1,0,0),(1,1,0),(2,1,0),(3,1,0)$, $(0,1,0),(-1,1,0),(-1,2,0),(-2,1,0),(-1,2,0),(-1,3,0), \quad(-$ $3,1,0),(-1,3,0)$ (again, all vectors are in units of grid points in the simulation box), and the vector $(0,0,1)$ for the translations in $z$ direction. Once all structure functions were calculated, the correlation functions are obtained by averaging over the 12 directions in the $\hat{\mathbf{x}}-\hat{\mathbf{y}}$ plane, and the parallel structure functions can be computed directly using the generator in the $\hat{\mathbf{z}}$ direction. In other words, the $\mathrm{SO}(2)$ decomposition gives the correlation functions along 12 polar directions in the $\hat{\mathbf{x}}-\hat{\mathbf{y}}$ plane and after averaging, one obtains a final correlation function as a function of the perpendicular polar direction (i.e., $\ell_{\perp}$ ) while $\mathrm{R}$ corresponds to the correlation function along the $\hat{\mathbf{z}}$ direction (i.e., with spatial increments $\ell_{\|}$) [49].

It is worth mentioning that Eq. (9) is valid for the mean values and not for each particular direction. In each of these decompositions we thus average the 73 (or 12) correlation functions of each term in Eq. (9) to investigate their relative importance in the compressible energy cascade rate. Although the $\mathrm{SO}(3)$ decomposition is better suited for isotropic turbulence, it has been used before to investigate anisotropic turbulence for the analysis of experimental results $[50,51]$ and numerical simulations $[48,52-54]$. The $\mathrm{SO}(2) \times \mathrm{R}$ decomposition, designed specifically from the symmetry group of axisymmetric turbulence, has been developed and used to investigate anisotropic turbulence using numerical simulations in [49]. In all cases, an improvement in the statistical convergence of correlation functions was observed when compared with correlation functions computed in only a few directions. 

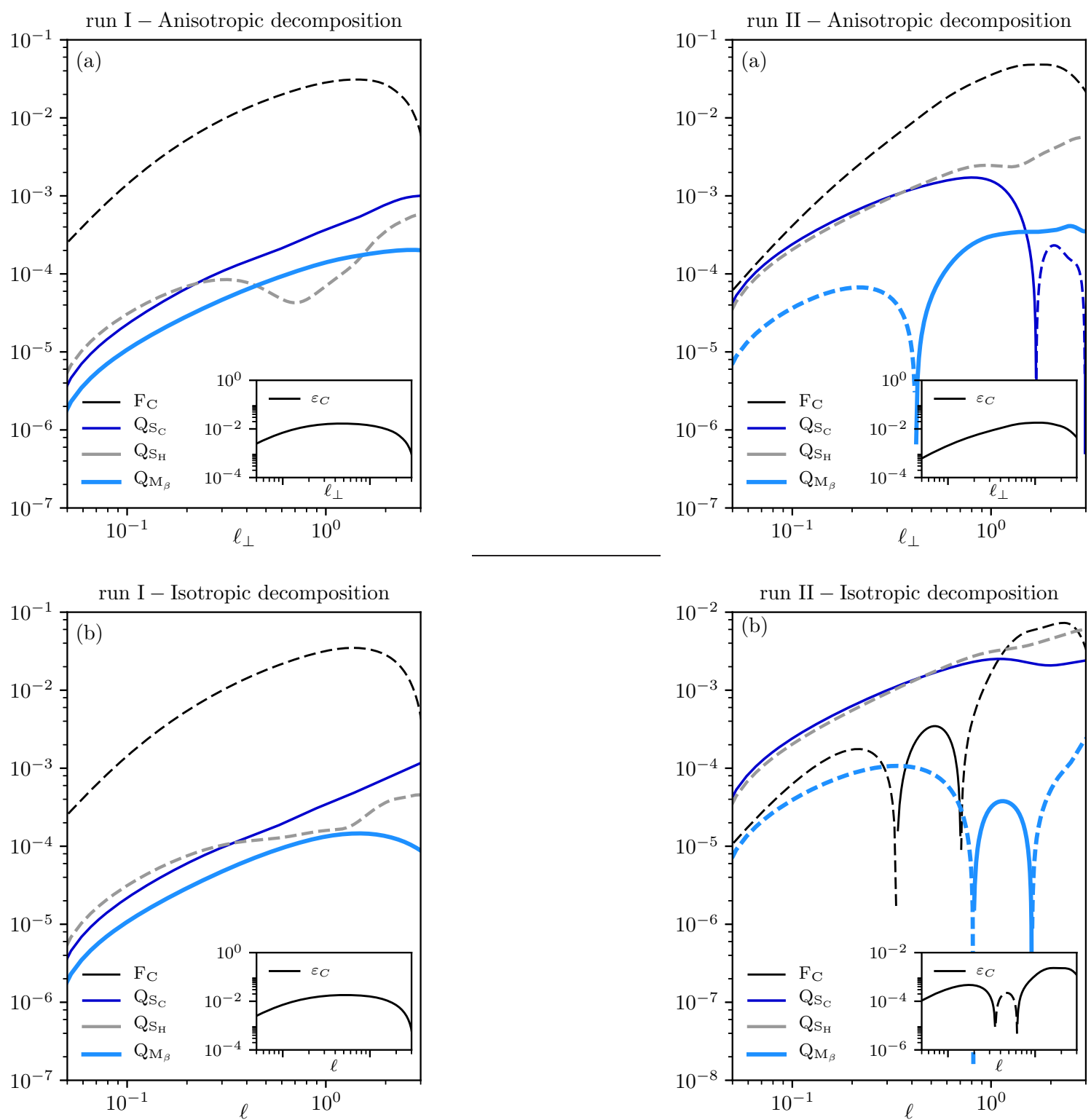

Figure 1. Run I: $B_{0}=0$ and $M_{S}=0.25$. Mean value of the compressible flux $\mathrm{F}_{\mathrm{C}}$ (black), source $\mathrm{S}_{\mathrm{C}}$ (dark blue), hybrid $\mathrm{S}_{\mathrm{H}}$ (gray) and $\beta$-dependent $\mathrm{M}_{\beta}$ (light blue) terms of the exact law (19) computed using the anisotropic (a) and isotropic (b) decompositions. Solid lines correspond to positive values while dashed lines correspond to negative values. Inset: total energy cascade rate computed using Eq. (19).

\section{NUMERICAL RESULTS}

For all runs in Table I, we computed the terms in the RHS of the exact law (9) using both the anisotropic and the isotropic decomposition techniques presented in Sec. II C. We investigate the different components and the
Figure 2. Run II: $B_{0}=2$ and $M_{S}=0.25$. Same description as in Fig. 1 applies.

energy cascade rate as we vary the sonic Mach number and the magnetic guide field in our simulations.

Figures 1(a) and 1(b) show for Run I the terms in the RHS of Eq. (19) as a function of the perpendicular $\left(\ell_{\perp}\right)$ and the isotropic $(\ell)$ scale obtained using the anisotropic and isotropic decomposition, respectively. Since there is no privileged direction in Run I $\left(B_{0}=\left|\mathbf{B}_{0}\right|=0\right)$, we find approximately the same variation and amplitude for the different terms as well as for the total energy cascade rate, independently of the decomposition used. 

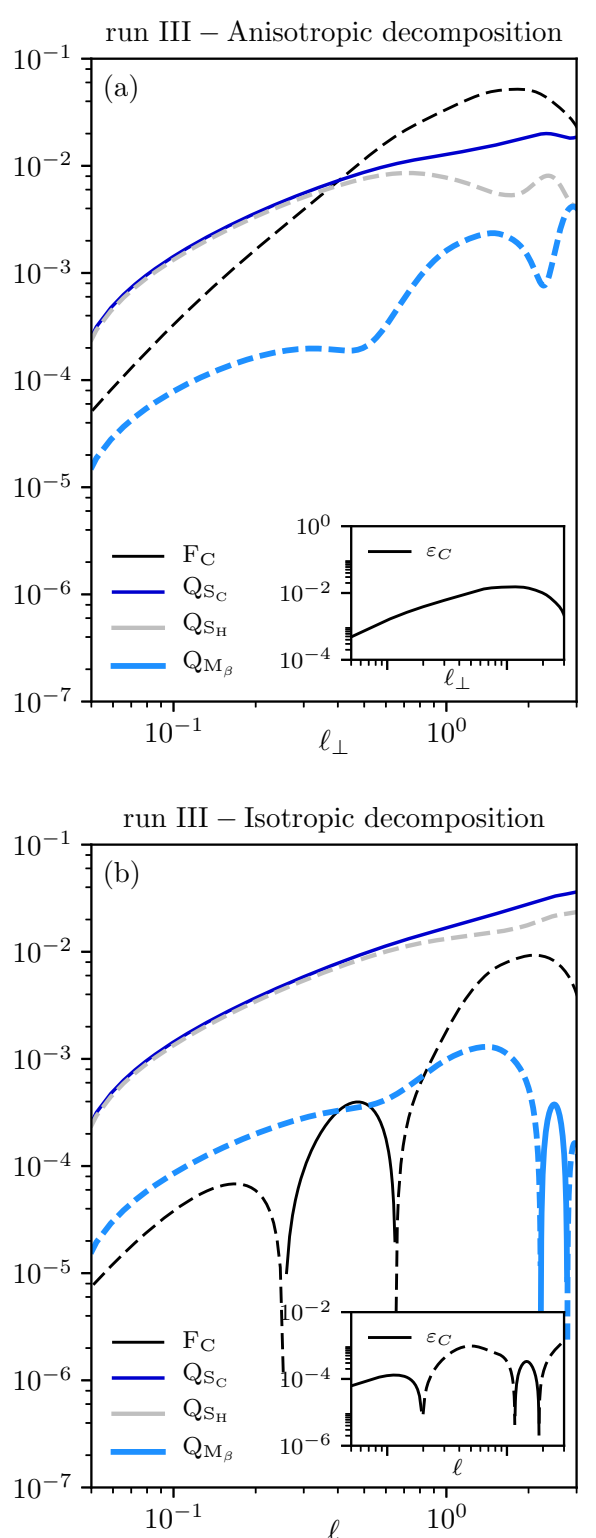

Figure 3. Run III: $B_{0}=8$ and $M_{S}=0.25$. Same description as in Fig. 1 applies.

There are indications of a fully developed turbulence regime that is compatible with a Kolmogorov-like scaling $[2,7,11,25,55]$ and with a constant energy cascade rate (see inset in Fig. 1). Note that at this moderate spatial resolution we cannot expect a wide inertial range. Nevertheless, the one evidenced here is sufficient for a first quantitative study of the different contributions to the exact law.

In the same format as Fig. 1, Figs. 2 and 3 display the results for Runs II and III respectively. As expected, the presence of a magnetic guide field $B_{0}$ strongly affects the statistical results. First, the compressible flux decreases slightly when $B_{0}$ is applied. We also see the appearance of a negative contribution (for Runs II and III) when the isotropic decomposition is used; this disrupts the scaling law that emerges. A comparison with the anisotropic decomposition reveals that the disruptions are a spurious effect due to the assumption of isotropy, which is not fulfilled in the runs with moderate to strong magnetic guide field [e.g., see 56]. Second, we find an increase of the source, hybrid and $\beta$-dependent (although in this case it is less important) integral terms when the magnetic guide field increases. For Run III, the source and hybrid terms become even dominant (in absolute value) at small scales; however, since they have the same amplitude but with a different sign they cancel each other leaving the compressible flux as the main contribution to the cascade rate. Still for Run III, it is interesting to note that it is precisely when the compressible flux dominates (in absolute value) that the source and hybrid terms behave differently. Finally, we see that the compressible cascade rate $\varepsilon_{C}$ is more difficult to evaluate in presence of $B_{0}$ because the inertial range becomes narrower (a higher spatial resolution seems to be necessary to get a reliable evaluation of this quantity). Note that in this case the fluctuating kinetic and magnetic energies become smaller (by a factor of $\sim 3$ ) in comparison with the cases without guide field, resulting from the fact that we kept the forcing amplitude fixed for all simulations independently of the value of $B_{0}$.

\section{A. Flux term}

The compressible flux in expression (15) can be decomposed as $\mathbf{F}_{\mathrm{C}}=\mathbf{F}_{1 a}+\mathbf{F}_{1 b}+\mathbf{F}_{2}$ with

$$
\begin{aligned}
\mathbf{F}_{1 a} & \equiv\left\langle\left[\left(\delta(\rho \mathbf{u}) \cdot \delta \mathbf{u}+\delta\left(\rho \mathbf{u}_{\mathrm{A}}\right) \cdot \delta \mathbf{u}_{\mathrm{A}}\right] \delta \mathbf{u}\right\rangle\right. \\
\mathbf{F}_{1 b} & \equiv-\left\langle\left[\delta(\rho \mathbf{u}) \cdot \delta \mathbf{u}_{\mathrm{A}}+\delta \mathbf{u} \cdot \delta\left(\rho \mathbf{u}_{\mathrm{A}}\right)\right] \delta \mathbf{u}_{\mathrm{A}}\right\rangle \\
\mathbf{F}_{2} & \equiv 2\langle\delta e \delta \rho \delta \mathbf{u}\rangle
\end{aligned}
$$

The term $\mathbf{F}_{1}=\mathbf{F}_{1 a}+\mathbf{F}_{1 b}$ can be identified as the compressible version of the (incompressible) MHD Yaglom flux [18] and $\mathbf{F}_{2}$ corresponds to a new purely compressible flux. Figure 4 shows the total compressible flux $\mathrm{F}_{\mathrm{C}}$ and its components $\mathrm{F}_{1 a}, \mathrm{~F}_{1 b}$ and $\mathrm{F}_{2}$ as a function of $\ell_{\perp}$ 


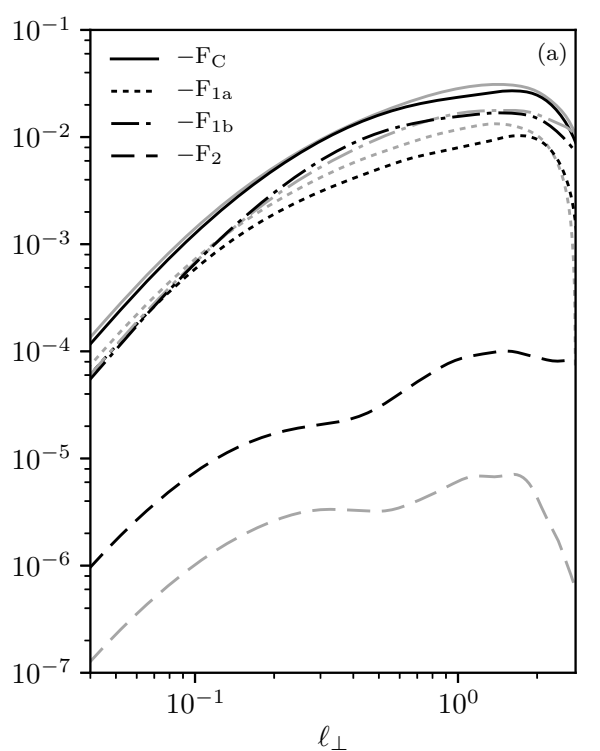

Figure 4. (a) Total compressible flux $\mathrm{F}_{\mathrm{C}}$ (solid) and its components $\mathrm{F}_{1 a}$ (dashed-dot), $\mathrm{F}_{1 b}$ (dot) and $\mathrm{F}_{2}$ (dashed) as a function of $\ell_{\perp}$, for Runs I (gray) and IV (black).
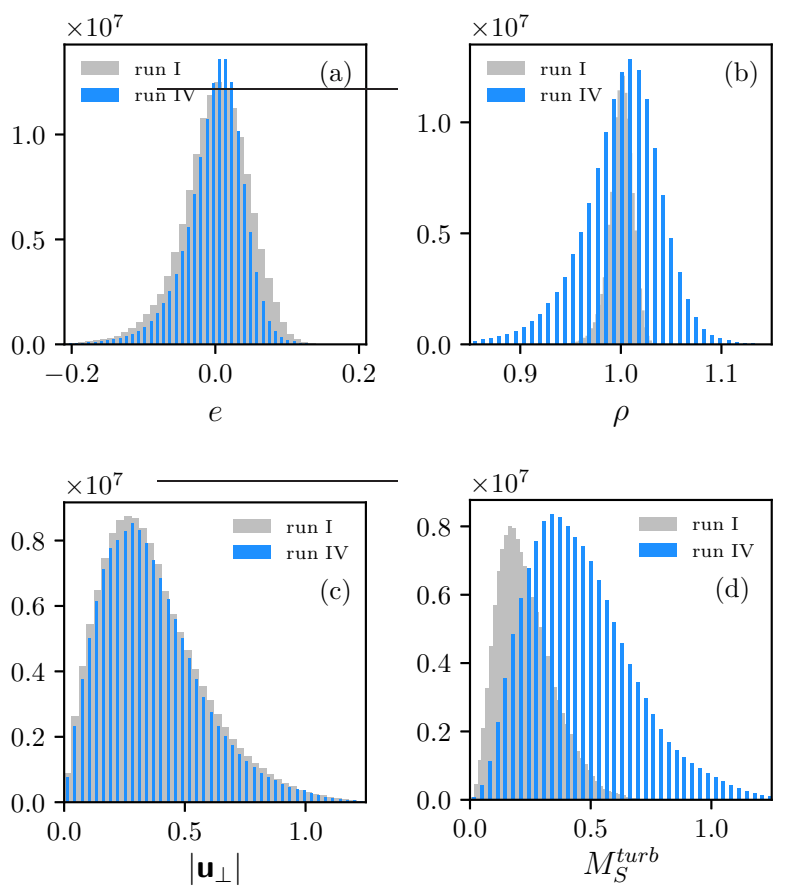

Figure 5. Histograms of the internal energy $e$ (a), the mass density $\rho(\mathrm{b})$, the absolute value of the perpendicular velocity $\left|\mathbf{u}_{\perp}\right|$ (c) and the turbulent sonic Mach number $M_{S}^{\text {turb }}$ (d), for runs I (grey) and IV (black lines). for Runs I and IV $\left(B_{0}=0\right)$ for $M_{S}=0.25$ and $M_{S}=0.5$ respectively. Figure 5 displays the histograms over all the numerical domain of the internal compressible energy $e$, density values $\rho$, the absolute value of the perpendicular velocity $\left|\mathbf{u}_{\perp}\right|$, and the turbulent Mach number $M_{S}^{t u r b} \equiv u_{r m s} / c_{s}$ for Runs I and IV. In Fig. 4 one can see that as the sonic Mach number increases the purely compressible component $\mathrm{F}_{2}$ experiences a strong increase (at least one order of magnitude through all spatial perpendicular scales), while the Yaglom-like terms $\mathrm{F}_{1 a}$ and $\mathrm{F}_{1 b}$ remain approximately the same. Furthermore, while $e$ and $\left|\mathbf{u}_{\perp}\right|$ have almost the same statistical values for both runs, the distribution of density values for $M_{S}=0.5$ has a larger spread around the reference density value $\left(\rho_{0}=1\right)$ than the one for $M_{S}=0.25$. In particular, we obtain a Gaussian distribution for the internal energy $e$, which is compatible with previous results in the literature [e.g., see 57-60]. Note that the internal energy is a quantity that is relevant for star formation dynamics [61]. The large spread in density values plus the different turbulent Mach numbers in both runs explain the strong increase in amplitude of $\mathrm{F}_{2}$. However, we see that even for $M_{S}=0.5$ the contribution of $\mathrm{F}_{2}$ to the total compressible flux remains negligible, which may be explained by the relatively low density fluctuations $\delta \rho / \rho \lesssim 10 \%$ as can be seen in Fig. 5 (b). Therefore, for small initial values of the sonic Mach number and zero magnetic guide field, the dominant contribution in the total compressible flux is due to the Yaglom-like terms.

Finally, we recall that in the present runs we used a solenoidal mechanical forcing for the velocity field. In runs with a balanced solenoidal/compressible external forcing, one may expect to obtain different results. This issue is particularly relevant in distant astrophysical plasmas such as the interstellar medium or supernova remnants $[58,62]$.

\section{B. Source, hybrid and $\beta$-dependent terms}

The source, hybrid and $\beta$-dependent terms of the exact law (9) arise exclusively because of the compressibility of the plasma [30] (in the incompressible case they are exactly null). In particular, while the source and hybrid terms are proportional to $\boldsymbol{\nabla} \cdot \mathbf{u}, \boldsymbol{\nabla} \cdot \mathbf{u}_{\mathrm{A}}$ and $\boldsymbol{\nabla} \cdot(\rho \mathbf{u})$, the 
run I

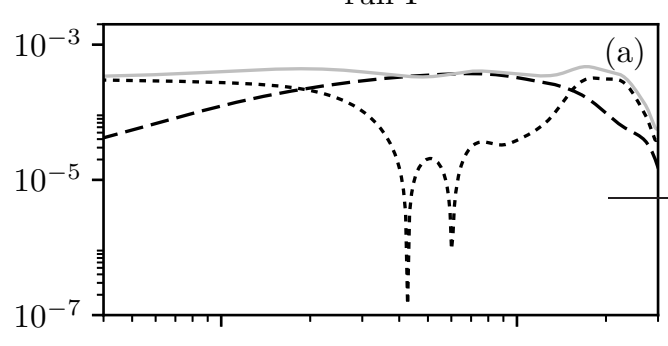

run II

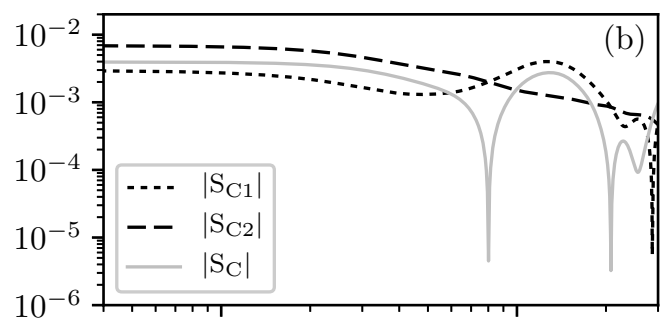

run III

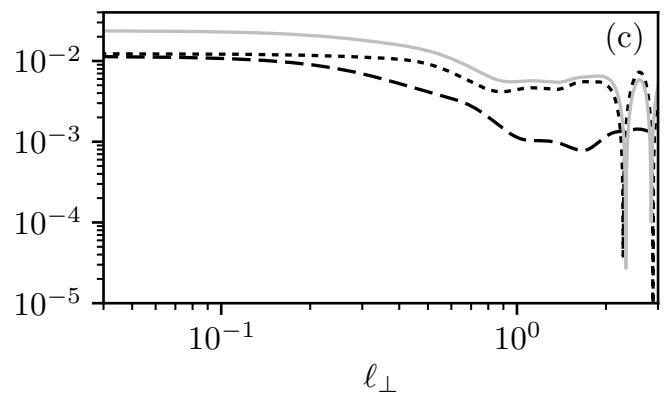

Figure 6. Total source term $\mathrm{S}_{\mathrm{C}}$ (light gray) and its components $\mathrm{S}_{\mathrm{C} 1}$ (dot black) and $\mathrm{S}_{\mathrm{C} 2}$ (dashed black) as a function of $\ell_{\perp}$ for Runs I (a), II (b) and III (c).

mixed $\beta$-dependent term is proportional to $\boldsymbol{\nabla} \cdot\left(e^{\prime} \rho \mathbf{u}\right)$. All these terms may modify the energy cascade rate in the inertial range, which is assumed to be constant at those scales.

The source (16) can be cast as $\mathrm{S}_{\mathrm{C}}=\mathrm{S}_{\mathrm{C} 1}+\mathrm{S}_{\mathrm{C} 2}$, with

$$
\begin{aligned}
\mathrm{S}_{\mathrm{C} 1} \equiv & \left\langle\left[R_{E}^{\prime}-\frac{1}{2}\left(R_{B}^{\prime}+R_{B}\right)\right](\boldsymbol{\nabla} \cdot \mathbf{u})\right. \\
& \left.+\left[R_{E}-\frac{1}{2}\left(R_{B}+R_{B}^{\prime}\right)\right]\left(\nabla^{\prime} \cdot \mathbf{u}^{\prime}\right)\right\rangle, \\
\mathrm{S}_{\mathrm{C} 2} \equiv & \left\langle\left[\left(R_{H}-R_{H}^{\prime}\right)-\bar{\rho}\left(\mathbf{u}^{\prime} \cdot \mathbf{u}_{\mathrm{A}}\right)\right]\left(\boldsymbol{\nabla} \cdot \mathbf{u}_{\mathrm{A}}\right)\right. \\
& \left.+\left[\left(R_{H}^{\prime}-R_{H}\right)-\bar{\rho}\left(\mathbf{u} \cdot \mathbf{u}_{\mathrm{A}}^{\prime}\right)\right]\left(\boldsymbol{\nabla}^{\prime} \cdot \mathbf{u}_{\mathrm{A}}^{\prime}\right)\right\rangle,
\end{aligned}
$$

where $\mathrm{S}_{\mathrm{C} 1}$ and $\mathrm{S}_{\mathrm{C} 2}$ correspond to the terms proportional to $\boldsymbol{\nabla} \cdot \mathbf{u}$ and $\boldsymbol{\nabla} \cdot \mathbf{u}_{\mathrm{A}}$, respectively. The hybrid term (17) (which can be expressed as a source or flux-like term
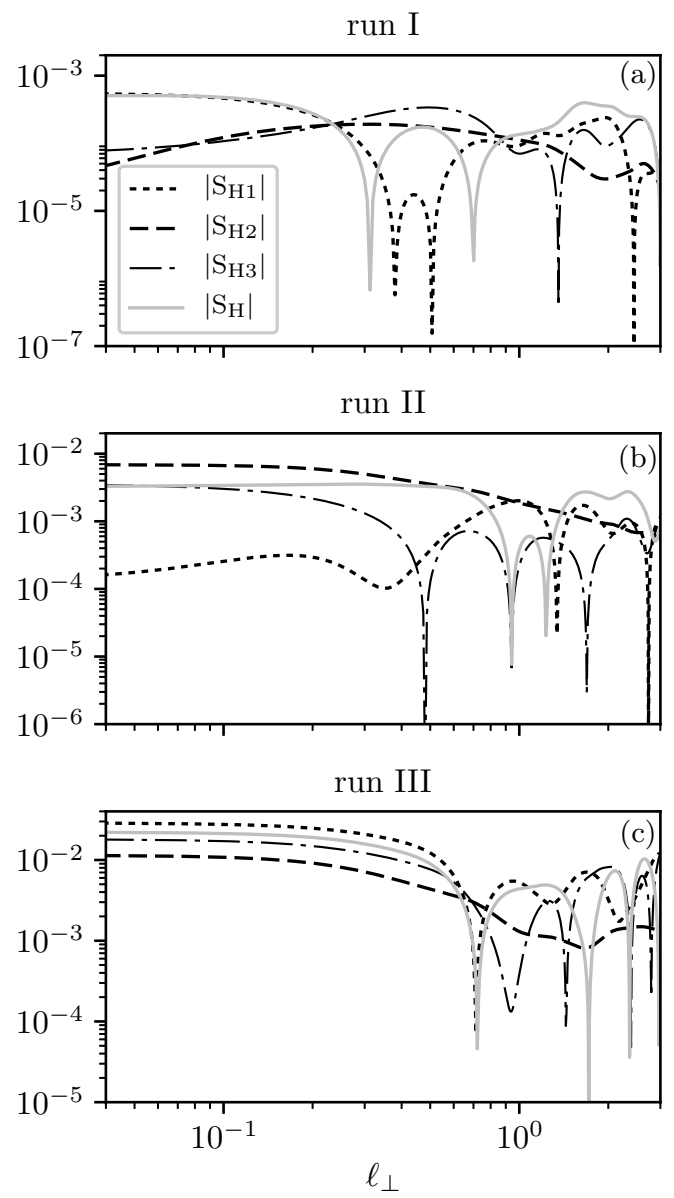

Figure 7. Total hybrid term $\mathrm{S}_{\mathrm{H}}$ (light gray) and its components $\mathrm{S}_{\mathrm{H} 1}$ (dot black), $\mathrm{S}_{\mathrm{H} 2}$ (dashed black) and $\mathrm{S}_{\mathrm{H} 3}$ (dasheddot black) as a function of $\ell_{\perp}$ for Runs I (a), II (b) and III (c).

$[16,20])$ can be cast as $\mathrm{S}_{\mathrm{H}}=\mathrm{S}_{\mathrm{H} 1}+\mathrm{S}_{\mathrm{H} 2}+\mathrm{S}_{\mathrm{H} 3}$, with

$\mathrm{S}_{\mathrm{H} 1} \equiv\left\langle\left(\frac{P_{M}^{\prime}-P^{\prime}}{2}-E^{\prime}\right)(\boldsymbol{\nabla} \cdot \mathbf{u})+\left(\frac{P_{M}-P}{2}-E\right)\left(\nabla^{\prime} \cdot \mathbf{u}^{\prime}\right)\right\rangle$,

$\mathrm{S}_{\mathrm{H} 2} \equiv\left\langle H^{\prime}\left(\boldsymbol{\nabla} \cdot \mathbf{u}_{\mathrm{A}}\right)+H\left(\boldsymbol{\nabla}^{\prime} \cdot \mathbf{u}_{\mathrm{A}}^{\prime}\right)\right\rangle$,

$\mathrm{S}_{\mathrm{H} 3} \equiv \frac{1}{2}\left\langle\left(e^{\prime}+\frac{u_{A}^{\prime 2}}{2}\right)[\nabla \cdot(\rho \mathbf{u})]+\left(e+\frac{u_{A}^{2}}{2}\right)\left[\nabla^{\prime} \cdot\left(\rho^{\prime} \mathbf{u}^{\prime}\right)\right]\right\rangle$,

where $\mathrm{S}_{\mathrm{H} 1}, \mathrm{~S}_{\mathrm{H} 2}$ and $\mathrm{S}_{\mathrm{H} 3}$ correspond to the terms proportional to $\boldsymbol{\nabla} \cdot \mathbf{u}, \boldsymbol{\nabla} \cdot \mathbf{u}_{\mathrm{A}}$ and $\boldsymbol{\nabla} \cdot(\rho \mathbf{u})$, respectively. Note that in recent observational works $[22,23]$, only the component $\mathrm{S}_{\mathrm{H} 3}$ was used to compute the solar wind energy cascade rate, besides the flux terms of Eq. 15. The rest of the hybrid components, i.e., $\mathrm{S}_{\mathrm{H} 1}$ and $\mathrm{S}_{\mathrm{H} 2}$, were assumed 

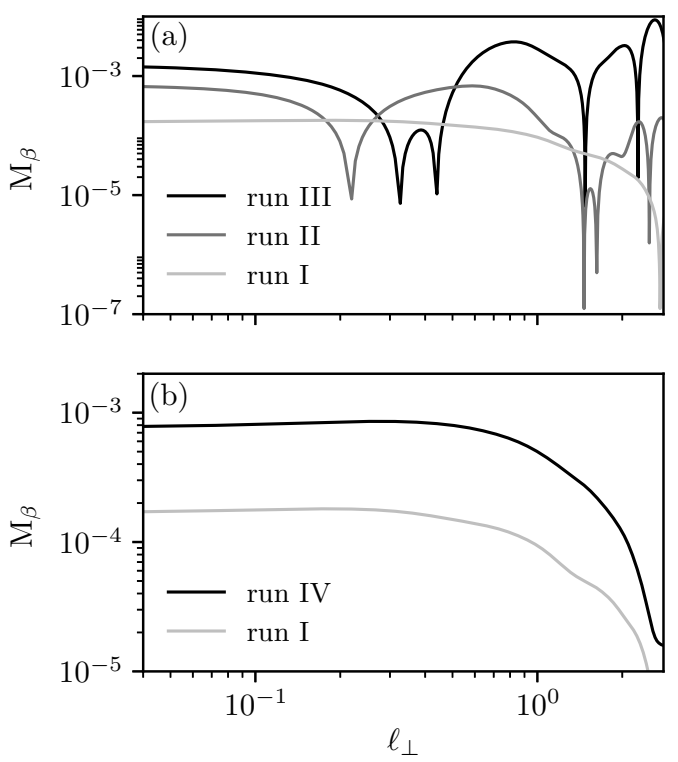

Figure 8. $\beta$-dependent terms $\mathrm{M}_{\beta}$ as a function of $\ell_{\perp}$ for (a) Runs I (light gray), II (gray) and III (black), and for (b) Run I (light gray) and IV (black), respectively.

to be sub-dominant in the inertial range. We will return to this point in Sec. IV.

Figures 6 and 7 show the absolute values of the source and hybrid terms as a function of $\ell_{\perp}$ for Runs I, II and III. Like above, the total (integrated) source and hybrid terms increase with increasing magnetic guide field (but while keeping the sonic Mach number constant). This behavior reflects the fact that $\mathrm{S}_{\mathrm{C}}$ and $\mathrm{S}_{\mathrm{H}}$ are explicitly proportional to $\mathbf{B}_{0}$ since $\mathbf{u}_{\mathrm{A}}$ includes the mean plus the fluctuations of the magnetic field. Furthermore, both terms tend to the same value in the small-scale limit.

Under the assumption of statistical stationarity of the $\beta$ parameter, the $\beta$-dependent term (18) can be converted into flux-like and be more easily measured using single-spacecraft data [see, 20, 22, 23]. However, in the present paper, we do not assume such additional hypothesis about the $\beta$ parameter. Figure 8 (a) displays the total $\beta$-dependent term $\mathrm{M}_{\beta}$ as a function of $\ell_{\perp}$ for $B_{0}=0$, $B_{0}=2$ and $B_{0}=8$ with $M_{S}=0.25$ (i.e., Runs I, II and III respectively) while Fig. 8(b) shows the same quantity for $M_{S}=0.25$ and $M_{S}=0.5$ with $B_{0}=0$ (Runs I and IV respectively). As for the other contributions, when we increase the magnetic guide field, the $\beta$-dependent term increases. We see, however, that it remains mainly smaller than the other contributions and in particular smaller than the compressible flux, which is compatible with the analysis shown in Figs. 1 to 3 . Finally, from Fig. 8(b) we note that this term has a strong dependence on the Mach number, as does the mass density fluctuations (see Fig. 5). This can be also concluded directly from Eq. (18). Note that in contrast to previous results [22], here we consider the total density values, i.e., the mean plus the fluctuation part.

\section{DISCUSSION AND CONCLUSION}

We have presented a first detailed 3D numerical analysis of the exact law for fully developed isothermal CMHD turbulence [16, 20]. Following Andrés and Sahraoui [20], we have separated the different contributions of the exact law in four types of terms, i.e., the compressible flux, source, hybrid and $\beta$-dependent terms. We run different simulations with varying initial Mach number and magnetic guide field. For all the runs, the compressible flux was found to be the dominant component in the exact law for CMHD turbulence. Furthermore, and as expected, this term is not strongly affected by the presence of a magnetic guide field $\mathbf{B}_{0}$ since it is a product of increments (and because the total density does not vary significantly between two points in space). In contrast, $\mathbf{B}_{0}$ was found to have a strong impact on the remaining terms of the exact law (9) [see also, 20] and also on the anisotropy of the flow $[30,56,63-72]$. Our numerical findings show a clear increase in $\mathrm{S}_{\mathrm{C}}, \mathrm{S}_{\mathrm{H}}$ and $\mathrm{M}_{\beta}$ terms as $B_{0}$ is increased from 0 to 8 . However, in all these cases the addition of these terms remain negligible with respect to the total compressible flux. Therefore, our energy cascade rate estimate has only a weak dependence on the magnetic guide field. It is worth mentioning that this result may be quite different if we consider the case of a strong guide field $\left(B_{0}>10\right)$, a supersonic turbulence $\left(M_{S}>1\right)$ and/or compressive driving of the velocity field.

Using in-situ measurements from the THEMIS mission, Banerjee et al. [23] and Hadid et al. [22] have investigated the role of compressible fluctuations in the MHD energy cascade rate for the fast and slow solar winds. Those works were extended recently to the terrestrial 
magnetosheath where a first estimation of the energy cascade rate is obtained [24]. The authors computed some of the terms of the exact law (9) and compared their relative impact on the total compressible energy cascade rate $\varepsilon_{C}$. In these original works, the authors used an isotropic decomposition to compute the Yaglom-like term (i.e., $\mathbf{F}_{1}$ ), the compressible flux (i.e., $\mathbf{F}_{2}$ ) and a third flux-like term $\mathbf{F}_{3}$, which is a combination of a part of the hybrid and the $\beta$-dependent (assuming statistical stationarity of $\beta$ ) terms. In particular,

$$
\mathbf{F}_{3}=2\left\langle\left(\bar{e}+\overline{\beta^{-1} e}+\frac{\overline{u_{\mathrm{A}}^{2}}}{2}\right) \delta\left(\rho_{1} \mathbf{u}\right)\right\rangle,
$$

where $\rho_{1}$ corresponds to the density fluctuations (the part proportional to $\rho_{0}$ has been written as a source and has not been computed). It is straightforward to identify the parts of $\mathrm{S}_{\mathrm{H} 3}$ and $\mathrm{M}_{\beta}$ which are involved in Eq. (29).

In Refs. [22, 23], the authors have found for the majority of the analyzed events comparable values of the compressible energy cascade rate $\varepsilon_{C}$ and the incompressible one $\varepsilon_{I}$ (computed from the exact law for IMHD turbulence $[5,6])$. That statistical result is compatible with our numerical findings, in which the Yaglom-like flux is the dominant component of Eq. (9) and is very close to the incompressible Yaglom term [18]. However, some of the spacecraft observations showed that the compressible Yaglom flux and/or the $\left(\mathbf{F}_{2}+\mathbf{F}_{3}\right)$ term can play a leading role in amplifying $\varepsilon_{C}$ with respect to $\varepsilon_{I}$, in particular in the slow solar wind (see Fig. 10 in Hadid et al. [22]). There are two possible explanations to those situations, which are not necessarily mutually exclusive. First, those events have larger density (and magnetic field) fluctuations that go beyond the values covered by our simulations in particular in the slow solar wind where $\delta \rho / \rho \lesssim 20 \%$ and the turbulent Mach number $M_{S}{ }^{\text {turb }} \lesssim 0.8$. This should be particularly true for the events that showed higher ratio of $\mathbf{F}_{1} / \mathbf{F}_{I}$ up to 10 (see Fig. 10 in [22]). The other possibility is that some missing (source) terms would have compensated (at least partly) the $\mathbf{F}_{3}$ term in those works, as we showed in the present simulations. Indeed, as recalled above, the observational results in Refs. [22-24] considered only the contributions from $\mathrm{S}_{\mathrm{H} 3}$, while our simulations results indicate that the other terms, $\mathrm{S}_{\mathrm{H} 1}$ and $\mathrm{S}_{\mathrm{H} 2}$, may well have equal contribution, and consequently should be considered. As we mentioned in Sec. III B, the compressible source terms involve local divergences that cannot be computed reliably using a single spacecraft because of the entanglement of the space and time variations (see Eq. 16). Thus, in Refs. [22-24], the authors had to assume that those terms are sub-dominant in the inertial range (this was also based on numerical simulations of supersonic hydrodynamic turbulence [41]). A future improvement of those observational works would be to try to estimate the missing (source and hybrid) terms using multispacecraft data from the Cluster or the MMS mission to evaluate the local vector field divergences. However, such methods remain to be developed. From the numerical viewpoint, the improvement of the present work would consist in making the code capable of capturing higher density fluctuations and higher Mach numbers than those studied here. This is needed to meet the physical conditions observed in particular in planetary magnetosheaths [24]. These problems will be investigated in forthcoming works.

\section{ACKNOWLEDGMENTS}

N.A. is supported through a DIM-ACAV postdoctoral fellowship and by LABEX Plas@Par through a grant managed by the Agence Nationale de la Recherche (ANR), as part of the program "Investissements d'Avenir" under the reference ANR-11-IDEX0004-02. F.S., S.G. and N.A. acknowledge financial support from Programme National Soleil-Terre (PNST). P.D. and P.D.M. acknowledge support from UBACYT Grant No. 20020130100738BA and PICT Grant No. 2015-3530. This work was granted access to the HPC resources of CINES under allocation 2017 A0030407714 made by GENCI. NA acknowledges Luis N. Martin for the useful discussions.
[1] U. Frisch, Turbulence: The Legacy of A. N. Kolmogorov (Cambridge University Press., 1995).
[2] A. N. Kolmogorov, Akademiia Nauk SSSR Doklady 30, 301 (1941a). 
[3] A. N. Kolmogorov, C.R. Acad. Sci. 32, 16 (1941b).

[4] S. Chandrasekhar, Proc. R. Soc. London, Ser A 204, 435 (1951).

[5] H. Politano and A. Pouquet, Phys. Rev. E 57, R21 (1998a).

[6] H. Politano and A. Pouquet, Geophys. Res. Lett. 25, 273 (1998b).

[7] P. D. Mininni and A. Pouquet, Phys. Rev. E 80, 025401 (2009).

[8] A. Bhattacharjee, Y.-M. Huang, H. Yang, and B. Rogers, Phys. Plasmas 16, 112102 (2009).

[9] M. Wan, S. Servidio, S. Oughton, and W. H. Matthaeus, Phys. Plasmas 17, 052307 (2010).

[10] K. Yoshimatsu, Phys. Rev. E 85, 066313 (2012).

[11] N. Andrés, P. Mininni, P. Dmitruk, and D. O. Gómez, Phys. Rev. E 93, 063202 (2016a).

[12] L. Sorriso-Valvo, R. Marino, V. Carbone, A. Noullez, F. Lepreti, P. Veltri, R. Bruno, B. Bavassano, and E. Pietropaolo, Phys. Rev. Lett. 99, 115001 (2007).

[13] J. M. Weygand, W. H. Matthaeus, S. Dasso, M. G. Kivelson, and R. J. Walker, J. Geophys. Res.: Space Phys. 112, A10 (2007).

[14] R. Marino, L. Sorriso-Valvo, V. Carbone, A. Noullez, R. Bruno, and B. Bavassano, Astrophys. J. Lett. 677 (2008).

[15] F. Sahraoui, Phys. Rev. E 78, 026402 (2008).

[16] B. Banerjee and S. Galtier, Phys. Rev. E 87, 013019 (2013).

[17] S. Galtier and S. Banerjee, Phys. Rev. Lett. 107, 134501 (2011).

[18] A. S. Monin and A. M. Yaglom, Statistical Fluid Mechanics: Mechanics of Turbulence, Vol. 2 (Cambridge, MA: MIT Press., 1975).

[19] S. Banerjee and C. Federrath, Phys. Rev. E 97, 023107 (2018).

[20] N. Andrés and F. Sahraoui, Phys. Rev. E 96, 053205 (2017b).

[21] V. Carbone, R. Marino, L. Sorriso-Valvo, A. Noullez, and R. Bruno, Phys. Rev. Lett. 103, 061102 (2009).

[22] L. Z. Hadid, F. Sahraoui, and S. Galtier, ApJ 9, 838 (2017a).

[23] S. Banerjee, L. Z. Hadid, F. Sahraoui, and S. Galtier, The Astrophysical Journal Letters 829, L27 (2016).

[24] L. Hadid, F. Sahraoui, S. Galtier, and S. Huang, Phys. Rev. Lett. 120, 055102 (2018).

[25] G. Zank, L. Adhikari, P. Hunana, D. Shiota, R. Bruno, and D. Telloni, The Astrophysical Journal 835, 147 (2017a).
[26] G. P. Zank and W. H. Matthaeus, Physical review letters 64, 1243 (1990).

[27] G. P. Zank, S. Du, and P. Hunana, The Astrophysical Journal 842, 114 (2017b).

[28] Y. Yang, W. H. Matthaeus, Y. Shi, M. Wan, and S. Chen, Physics of Fluids 29, 035105 (2017).

[29] P. Grete, B. W. O'Shea, K. Beckwith, W. Schmidt, and A. Christlieb, Physics of Plasmas 24, 092311 (2017).

[30] N. Andrés, P. Clark di Leoni, P. D. Mininni, P. Dmitruk, F. Sahraoui, and W. H. Matthaeus, Physics of Plasmas 24, 102314 (2017a).

[31] N. Andrés, S. Galtier, and F. Sahraoui, Phys. Rev. E 97, 013204 (2018).

[32] R. Fitzpatrick, Plasma Physics: An Introduction (CRC Press, 2014).

[33] E. Marsch and A. Mangeney, J. Geophys. Res. 92, 7363 (1987).

[34] J. Duchon and R. Robert, Nonlinearity 13, 249-255 (2000).

[35] G. L. Eyink and T. D. Drivas, Phys. Rev. X 8, 011022 (2018).

[36] S. Galtier, J. Physics A: Mathematical \& Theoretical 51, 205501 (2018).

[37] N. Andrés, S. Galtier, and F. Sahraoui, Phys. Rev. E 94, 063206 (2016c).

[38] G. K. Batchelor, The theory of homogeneus turbulence (Cambridge Univ. Press, 1953).

[39] T. von Kármán and L. Howarth, Proc. R. Soc. London 164, 192 (1938).

[40] S. Banerjee and S. Galtier, Journal of Fluid Mechanics 742, 230 (2014).

[41] A. G. Kritsuk, R. Wagner, and M. L. Norman, Journal of Fluid Mechanics 729, R1 (2013).

[42] D. O. Gómez, P. D. Mininni, and P. Dmitruk, Phys. Scripta T116 123 (2005).

[43] P. D. Mininni, D. Rosenberg, R. Reddy, and A. Pouquet, Parallel Computing 37, 16 (2011).

[44] S. Ghosh, M. Hossain, and W. H. Matthaeus, Computer Phys. Commun. 74, 18 (1993).

[45] P. Dmitruk, W. H. Matthaeus, and S. Oughton, Phys. Plasmas 12, 112304 (2005).

[46] M. A. Taylor, S. Kurien, and G. L. Eyink, Physical Review E 68, 026310 (2003).

[47] L. Martin and P. Mininni, Physical Review E 81, 016310 (2010).

[48] I. Arad, L. Biferale, I. Mazzitelli, and I. Procaccia, Physical review letters 82, 5040 (1999).

[49] P. R. Imazio and P. Mininni, Physical Review E 95, 033103 (2017). 
[50] S. Kurien and K. R. Sreenivasan, Physical Review E 62, 2206 (2000a).

[51] S. Kurien, V. S. L'vov, I. Procaccia, and K. Sreenivasan, Physical Review E 61, 407 (2000b).

[52] L. Biferale and F. Toschi, Physical review letters 86, 4831 (2001).

[53] P. D. Mininni and A. Pouquet, Physics of Fluids 22, 035105 (2010).

[54] P. R. Imazio and P. Mininni, Physical Review E 83, 066309 (2011).

[55] W. H. Matthaeus and M. L. Goldstein, J. Geophys. Res. 87, 6011 (1982).

[56] C. Federrath, Journal of Plasma Physics 82, 535820601 (2016).

[57] T. Passot and E. Vázquez-Semadeni, Phys. Rev. E 58, 4501 (1998).

[58] C. Federrath, J. Roman-Duval, R. S. Klessen, W. Schmidt, and M.-M. Mac Low, A\&A 512, A 81 (2010).

[59] C. Federrath and S. Banerjee, Monthly Notices of the Royal Astronomical Society 448, 3297 (2015).

[60] C. A. Nolan, C. Federrath, and R. S. Sutherland, MNRAS 451, 1380 (2015).

[61] C. Federrath and R. S. Klessen, The Astrophysical Journal 761, 156 (2012).
[62] C. Federrath, J. M. Rathborne, S. N. Longmore, J. M. D. Kruijssen, J. Bally, Y. Contreras, R. M. Crocker, G. Garay, J. M. Jackson, L. Testi, and A. J. Walsh, in The Multi-Messenger Astrophysics of the Galactic Centre, IAU Symposium, Vol. 322, edited by R. M. Crocker, S. N. Longmore, and G. V. Bicknell (2017) pp. 123-128.

[63] J. V. Shebalin, W. H. Matthaeus, and D. Montgomery, Journal of Plasma Physics 29, 525 (1983).

[64] W. H. Matthaeus, S. Ghosh, S. Oughton, and D. A. Roberts, Journal of Geophysical Research: Space Physics 101, 7619 (1996).

[65] M.-M. Mac Low, Astrophys. J. 524, 169 (1999).

[66] S. Galtier, S. V. Nazarenko, A. C. Newell, and A. Pouquet, J. Plasma Phys. 63, 447 (2000).

[67] C. M. Brunt, C. Federrath, and D. J. Price, MNRAS 403, 1507 (2010).

[68] M. Wan, S. Oughton, S. Servidio, and W. H. Matthaeus, J. Fluid Mech. 697, 296 (2012).

[69] S. Oughton, M. Wan, S. Servidio, and W. H. Matthaeus, ApJ 768, 10 (2013).

[70] R. Meyrand, S. Galtier, and K. H. Kiyani, Phys. Rev. Lett. 116, 105002 (2016).

[71] S. Oughton, W. H. Matthaeus, M. Wan, and T. Parashar, J. Geophys. Res. 121, 5041 (2016).

[72] N. E. Sujovolsky and P. D. Mininni, Phys. Rev. Fluids 1, 054407 (2016). 